\title{
Sustainable production of bioplastics from lignocellulosic biomass: techno-economic analysis and life-cycle assessment
}

\author{
Hyunwoo Kim ${ }^{1 \dagger}$, Shinje Lee ${ }^{1 \dagger}$, Yuchan Ahn ${ }^{2 \dagger}$, Jinwon Lee ${ }^{3}$, and Wangyun Won ${ }^{1 *}$
}

1 Department of Chemical Engineering, Kyung Hee University,

1732 Deogyeong-daero, Giheung-gu, Yongin-si, Gyeonggi-do 17104, Republic of Korea

2 Green Materials \& Processes group, Korea Institute of Industrial Technology, 55 Jongga-ro, Jung-gu, Ulsan 44413, Republic of Korea

3 Department of Chemical and Biomolecular Engineering, Sogang University, 35 Baekbeom-ro, Mapo-gu, Seoul 04107, Republic of Korea

Corresponding author (E-mail: wwon@khu.ac.kr)

$\dagger$ Equally contributed

\section{Supporting Information}

Number of Pages: 19

Number of Figures: 6

Number of Tables: 12 


\section{Economic analysis}

Table S1. Major economic parameters and assumptions.

\begin{tabular}{|c|c|}
\hline${\text { Feedstock price }(\$ / \text { ton })^{a}}^{a}$ & 125.0 \\
\hline$\gamma$-valerolactone price $(\$ / \text { ton })^{\text {a }}$ & 1000.0 \\
\hline Sodium chloride price (\$/ton) ${ }^{a}$ & 182.0 \\
\hline Sulfuric acid price (\$/ton) ${ }^{b}$ & 110.0 \\
\hline Hydrogen price $(\$ / \text { ton })^{b}$ & 1507.5 \\
\hline Gypsum disposal cost (\$/ton) ${ }^{b}$ & 31.9 \\
\hline Cooling tower chemicals price $(\$ / \text { ton })^{b}$ & 3671.4 \\
\hline $\mathrm{Ca}(\mathrm{OH})_{2}$ price $(\$ / \text { ton })^{\mathrm{c}}$ & 221.9 \\
\hline Tetrahydrofuran price (\$/ton) ${ }^{\mathrm{c}}$ & 1532.0 \\
\hline Oxygen price $(\$ / \text { ton })^{d}$ & 40.0 \\
\hline $\mathrm{Pt} / \mathrm{C}$ catalyst price $(\$ / \mathrm{kg}){ }^{\mathrm{e}}$ & 194.0 \\
\hline Natural gas price $(\$ / \text { ton })^{\mathrm{f}}$ & 239.0 \\
\hline $\mathrm{RuSn}_{4} / \mathrm{C}$ catalyst price $(\$ / \mathrm{kg})^{\mathrm{g}}$ & 539.3 \\
\hline $\mathrm{Ru} / \mathrm{C}$ catalyst price $(\$ / \mathrm{kg})^{\mathrm{h}}$ & 88.0 \\
\hline Activated carbon price $(\$ / \text { ton })^{\mathrm{i}}$ & 1200.0 \\
\hline Plant financing by equity (\%) ${ }^{b}$ & 40.0 \\
\hline Plant life (years) ${ }^{b}$ & 30.0 \\
\hline Income tax rate $(\%)^{b}$ & 35.0 \\
\hline Interest rate for debt financing $(\%)^{b}$ & 8.0 \\
\hline Term for debt financing (years) ${ }^{\mathrm{b}}$ & 10.0 \\
\hline General plant depreciation (years) ${ }^{b}$ & 7.0 \\
\hline Steam plant depreciation (years) ${ }^{b}$ & 20.0 \\
\hline Working capital (\% of fixed capital investment) $b$ & 5.0 \\
\hline On-stream percentage after startup (\%) ${ }^{\mathrm{b}}$ & 90.0 \\
\hline Startup time (years) ${ }^{b}$ & 0.5 \\
\hline \multicolumn{2}{|l|}{ Revenue and costs during startup } \\
\hline Revenue (\% of normal) ${ }^{b}$ & 50.0 \\
\hline Variable costs ( $\%$ of normal) ${ }^{b}$ & 75.0 \\
\hline Fixed costs (\% of normal) ${ }^{b}$ & 100.0 \\
\hline Construction period (years) ${ }^{b}$ & 3.0 \\
\hline First 12 months' expenditures (\%) ${ }^{b}$ & 8.0 \\
\hline Next 12 months' expenditures (\%) ${ }^{b}$ & 60.0 \\
\hline Last 12 months' expenditures (\%) b & 32.0 \\
\hline
\end{tabular}

a Taken from Alonso et al. ${ }^{1}$

b Taken from Davis et al. ${ }^{2}$

c Taken from He et al. ${ }^{3}$

d Taken from Dorris et al. ${ }^{4}$

e Taken from Han et al. ${ }^{5}$

f Taken from Dutta et al. ${ }^{6}$

g Taken from Han et al. ${ }^{7}$

h Taken from Brentzel et al. ${ }^{8}$

i Taken from Motagamwala et al. ${ }^{9}$

Assumption:

- $10 \%$ of the catalyst is refurbished every 6 months at a cost equivalent to $20 \%$ of its original value. ${ }^{5}$

- Discount rate is assumed to be $20 \%$. 


\section{Important stream information}

Table S2. Process stream information.

\begin{tabular}{|c|c|c|c|c|c|c|c|c|c|c|c|c|c|c|c|c|c|}
\hline & 1 & 8 & 13 & 17 & 19 & 25 & 30 & 33 & 40 & 49 & 58 & 59 & 61 & 63 & 70 & 74 & 77 \\
\hline \multicolumn{18}{|c|}{$\begin{array}{l}\text { Components mass flow } \\
\text { (ton/hr) }\end{array}$} \\
\hline $\mathrm{H}_{2} \mathrm{O}$ & 35.7 & 0.0 & 44.0 & 0.0 & 0.0 & 0.0 & 0.0 & 4.1 & 0.0 & 112.8 & 3.6 & 0.0 & 70.3 & 0.0 & 67.5 & 64.8 & 66.4 \\
\hline GVL & 0.0 & 1.0 & 106.7 & 106.7 & 0.8 & 0.0 & 0.0 & 0.0 & 0.0 & 0.0 & 1.0 & 0.0 & 69.0 & 0.0 & 66.3 & 66.3 & 69.3 \\
\hline $\mathrm{NaCl}$ & 0.0 & 0.0 & 0.6 & 0.6 & 0.6 & 0.0 & 0.0 & 0.0 & 0.0 & 0.0 & 0.0 & 0.0 & 0.0 & 0.0 & 0.0 & 0.0 & 0.0 \\
\hline $\mathrm{H}_{2} \mathrm{SO}_{4}$ & 0.0 & 0.1 & 1.0 & 1.0 & 1.0 & 0.0 & 0.0 & 0.0 & 0.0 & 0.0 & 0.0 & 0.0 & 0.0 & 0.0 & 0.0 & 0.0 & 0.0 \\
\hline Acetic acid & 0.0 & 0.0 & 5.0 & 0.0 & 0.0 & 0.0 & 0.0 & 0.0 & 0.0 & 0.0 & 0.0 & 0.0 & 0.0 & 0.0 & 0.0 & 0.0 & 0.0 \\
\hline Furfural & 0.0 & 0.0 & 1.8 & 0.1 & 0.0 & 11.4 & 0.1 & 0.1 & 0.0 & 0.0 & 0.7 & 0.0 & 0.0 & 0.0 & 0.0 & 0.0 & 0.0 \\
\hline HMF & 0.0 & 0.0 & 0.1 & 0.1 & 0.1 & 0.0 & 0.0 & 0.0 & 0.0 & 0.0 & 11.4 & 0.0 & 0.0 & 0.0 & 0.0 & 0.0 & 0.0 \\
\hline Lignin & 18.1 & 1.2 & 16.9 & 16.9 & 16.9 & 0.0 & 0.0 & 0.0 & 0.0 & 0.0 & 1.2 & 0.0 & 0.0 & 0.0 & 0.0 & 0.0 & 0.0 \\
\hline Extract & 0.0 & 0.0 & 0.5 & 0.5 & 0.5 & 0.0 & 0.0 & 0.0 & 0.0 & 0.0 & 0.0 & 0.0 & 0.0 & 0.0 & 0.0 & 0.0 & 0.0 \\
\hline Glucose & 0.0 & 0.2 & 2.3 & 0.6 & 0.6 & 0.0 & 0.0 & 0.0 & 0.0 & 0.0 & 0.5 & 0.0 & 0.1 & 0.0 & 0.1 & 0.1 & 0.1 \\
\hline Xylose & 0.0 & 1.2 & 18.0 & 0.2 & 0.2 & 0.0 & 0.0 & 0.0 & 0.0 & 0.0 & 1.2 & 0.0 & 0.3 & 0.0 & 0.3 & 0.3 & 0.3 \\
\hline LA & 0.0 & 0.1 & 0.5 & 0.8 & 0.0 & 0.0 & 0.0 & 0.0 & 0.0 & 0.0 & 0.6 & 0.0 & 0.5 & 0.0 & 0.5 & 0.5 & 0.0 \\
\hline FA & 0.0 & 0.0 & 0.1 & 0.0 & 0.0 & 0.0 & 0.0 & 0.0 & 0.0 & 0.3 & 0.2 & 0.0 & 0.0 & 0.0 & 0.0 & 0.0 & 0.0 \\
\hline Heavy oil & 0.0 & 0.0 & 0.0 & 0.0 & 0.4 & 0.0 & 0.0 & 0.0 & 0.0 & 0.0 & 0.0 & 0.0 & 0.0 & 0.0 & 0.0 & 0.0 & 0.0 \\
\hline $\mathrm{CaSO}_{4}$ & 0.0 & 0.0 & 0.6 & 0.6 & 0.6 & 0.0 & 0.0 & 0.0 & 0.0 & 0.0 & 0.0 & 0.0 & 0.0 & 0.0 & 0.0 & 0.0 & 0.0 \\
\hline Ash & 0.2 & 0.0 & 0.0 & 0.0 & 0.0 & 0.0 & 0.0 & 0.0 & 0.0 & 0.0 & 0.0 & 0.0 & 0.0 & 0.0 & 0.0 & 0.0 & 0.0 \\
\hline Sextract & 0.6 & 0.0 & 0.0 & 0.0 & 0.0 & 0.0 & 0.0 & 0.0 & 0.0 & 0.0 & 0.0 & 0.0 & 0.0 & 0.0 & 0.0 & 0.0 & 0.0 \\
\hline Protein & 0.8 & 0.8 & 0.0 & 0.0 & 0.0 & 0.0 & 0.0 & 0.0 & 0.0 & 0.0 & 0.8 & 0.0 & 0.0 & 0.0 & 0.0 & 0.0 & 0.0 \\
\hline Acetate & 5.0 & 0.0 & 0.0 & 0.0 & 0.0 & 0.0 & 0.0 & 0.0 & 0.0 & 0.0 & 0.0 & 0.0 & 0.0 & 0.0 & 0.0 & 0.0 & 0.0 \\
\hline Cellulose & 37.5 & 34.9 & 0.0 & 0.0 & 0.0 & 0.0 & 0.0 & 0.0 & 0.0 & 0.0 & 0.0 & 0.0 & 0.0 & 0.0 & 0.0 & 0.0 & 0.0 \\
\hline Xylan & 21.1 & 1.9 & 0.0 & 0.0 & 0.0 & 0.0 & 0.0 & 0.0 & 0.0 & 0.0 & 1.9 & 0.0 & 0.0 & 0.0 & 0.0 & 0.0 & 0.0 \\
\hline Humins & 0.0 & 0.0 & 0.0 & 2.6 & 2.6 & 0.0 & 0.0 & 0.0 & 0.0 & 0.0 & 17.4 & 0.0 & 0.0 & 0.0 & 0.0 & 0.0 & 0.0 \\
\hline $\mathrm{H}_{2}$ & 0.0 & 0.0 & 0.0 & 0.0 & 0.0 & 0.0 & 0.0 & 0.0 & 0.0 & 0.0 & 0.0 & 0.0 & 0.0 & 0.0 & 0.0 & 0.0 & 0.0 \\
\hline $\mathrm{O}_{2}$ & 0.0 & 0.0 & 0.0 & 0.0 & 0.0 & 0.0 & 0.0 & 0.0 & 0.0 & 0.0 & 0.0 & 4.0 & 0.0 & 0.0 & 0.0 & 0.0 & 0.0 \\
\hline $\mathrm{CO}_{2}$ & 0.0 & 0.0 & 0.0 & 0.0 & 0.0 & 0.0 & 0.0 & 0.0 & 0.0 & 0.0 & 0.0 & 0.0 & 0.0 & 0.0 & 0.0 & 0.0 & 0.0 \\
\hline 2-MBA & 0.0 & 0.0 & 0.0 & 0.0 & 0.0 & 0.0 & 0.0 & 0.0 & 0.0 & 0.0 & 0.0 & 0.0 & 0.0 & 0.0 & 0.0 & 0.0 & 0.0 \\
\hline THF & 0.0 & 0.0 & 0.0 & 0.0 & 0.0 & 0.0 & 0.0 & 0.0 & 0.0 & 1016.3 & 0.0 & 0.0 & 0.0 & 0.0 & 0.0 & 0.0 & 0.0 \\
\hline Formaldehyde & 0.0 & 0.0 & 0.0 & 0.0 & 0.0 & 0.0 & 0.0 & 0.0 & 0.0 & 66.7 & 0.0 & 0.0 & 0.0 & 0.0 & 0.0 & 0.0 & 0.0 \\
\hline FDCA & 0.0 & 0.0 & 0.0 & 0.0 & 0.0 & 0.0 & 0.0 & 0.0 & 0.0 & 0.0 & 0.0 & 0.0 & 13.0 & 12.3 & 0.6 & 0.6 & 0.6 \\
\hline Levoglucosan & 0.0 & 0.0 & 0.0 & 0.0 & 0.0 & 0.0 & 0.0 & 0.0 & 0.0 & 0.0 & 0.0 & 0.0 & 0.0 & 0.0 & 0.0 & 0.0 & 0.0 \\
\hline Levoglucosenone & 0.0 & 0.0 & 0.1 & 0.1 & 0.1 & 0.0 & 0.0 & 0.0 & 0.0 & 0.0 & 0.6 & 0.0 & 4.3 & 0.0 & 4.1 & 4.1 & 4.3 \\
\hline Polymer & 0.0 & 0.0 & 0.0 & 0.0 & 0.0 & 0.0 & 0.0 & 0.0 & 0.0 & 0.0 & 0.0 & 0.0 & 1.1 & 0.0 & 1.1 & 1.1 & 1.1 \\
\hline THFA & 0.0 & 0.0 & 0.0 & 0.0 & 0.0 & 0.0 & 9.4 & 0.0 & 0.0 & 0.0 & 0.0 & 0.0 & 0.0 & 0.0 & 0.0 & 0.0 & 0.0 \\
\hline FFA & 0.0 & 0.0 & 0.0 & 0.0 & 0.0 & 0.0 & 0.7 & 0.7 & 0.0 & 0.0 & 0.0 & 0.0 & 0.0 & 0.0 & 0.0 & 0.0 & 0.0 \\
\hline DHP & 0.0 & 0.0 & 0.0 & 0.0 & 0.0 & 0.0 & 0.0 & 7.0 & 0.0 & 0.0 & 0.0 & 0.0 & 0.0 & 0.0 & 0.0 & 0.0 & 0.0 \\
\hline THP-OXY & 0.0 & 0.0 & 0.0 & 0.0 & 0.0 & 0.0 & 0.0 & 0.0 & 0.1 & 0.0 & 0.0 & 0.0 & 0.0 & 0.0 & 0.0 & 0.0 & 0.0 \\
\hline 2,2-HY-THP & 0.0 & 0.0 & 0.0 & 0.0 & 0.0 & 0.0 & 0.0 & 0.0 & 0.0 & 0.0 & 0.0 & 0.0 & 0.0 & 0.0 & 0.0 & 0.0 & 0.0 \\
\hline 2-HY-ТHP & 0.0 & 0.0 & 0.0 & 0.0 & 0.0 & 0.0 & 0.0 & 0.0 & 0.0 & 0.0 & 0.0 & 0.0 & 0.0 & 0.0 & 0.0 & 0.0 & 0.0 \\
\hline Coke & 0.0 & 0.0 & 0.0 & 0.0 & 0.0 & 0.0 & 0.0 & 0.0 & 0.0 & 0.0 & 0.0 & 0.0 & 0.0 & 0.0 & 0.0 & 0.0 & 0.0 \\
\hline 1,5-PDO & 0.0 & 0.0 & 0.0 & 0.0 & 0.0 & 0.0 & 0.0 & 0.0 & 8.0 & 0.0 & 0.0 & 0.0 & 0.0 & 0.0 & 0.0 & 0.0 & 0.0 \\
\hline
\end{tabular}


Activated carbon

Total mass flow (ton $/ \mathrm{hr}$ )

Temperature (K)

$\begin{array}{rrrrrr}0.0 & 0.0 & 0.0 & 0.0 & 0.0 & 0.0 \\ 0.0 & 0.0 & 0.0 & 0.0 & 0.0 & 0.0 \\ 0.0 & 0.0 & 0.0 & 0.0 & 0.0 & 0.0 \\ 119.0 & 41.6 & 198.1 & 130.7 & 24.3 & 11.4 \\ 298.1 & 413.8 & 398.4 & 496.4 & 495.2 & 440.9 \\ 1.0 & 1.0 & 20.4 & 1.3 & 1.2 & 1.2\end{array}$

$\begin{array}{rr}0.0 & \\ 0.0 & \\ 0.0 & 0.0 \\ 1.4 & 0.0 \\ 40.9 & 449.4 \\ 1.2 & 10.0\end{array}$

$\begin{array}{rr}0.0 & 0.0 \\ 0.0 & 0.0 \\ 0.0 & 0.0 \\ 10.2 & 11.9 \\ 449.4 & 345.2 \\ 1.0 & 1.0\end{array}$

$\begin{array}{rr}0.0 & \\ 0.0 & 0.0 \\ 0.0 & 0.0 \\ 11.9 & 8.1 \\ 45.2 & 484.2 \\ 1.0 & 0.4\end{array}$

$\begin{array}{rrr}0.0 & 0.0 & 0.0 \\ 0.0 & 0.0 & 0.0 \\ 0.0 & 0.0 & 0.0 \\ 8.1 & 1196.1 & 41.2 \\ 84.2 & 338.0 & 439.5 \\ 0.4 & 1.0 & 3.0\end{array}$

$\begin{array}{rr}0.0 & \\ 0.0 & 0.0 \\ 0.0 & 0.0 \\ 41.2 & 5.0 \\ 439.5 & 298.2\end{array}$

$\begin{array}{rr}0.0 & \\ 0.0 & 0.0 \\ 0.0 & 0.0 \\ 5.0 & 158.6 \\ 298.2 & 298.2\end{array}$

\begin{tabular}{rrrrr}
0.0 & 0.0 & 0.0 & 0.0 & 0.0 \\
0.0 & 0.0 & 0.0 & 0.0 & 0.0 \\
0.0 & 0.0 & 0.0 & 0.0 & 0.0 \\
158.6 & 12.4 & 140.5 & 137.8 & 142.3 \\
298.2 & 352.0 & 298.2 & 383.3 & 378.2 \\
\hline 0.0 & 1.0 & 40.0 & 1.2 & 1.0
\end{tabular}

Pressure (atm) 


\section{Heat integration}

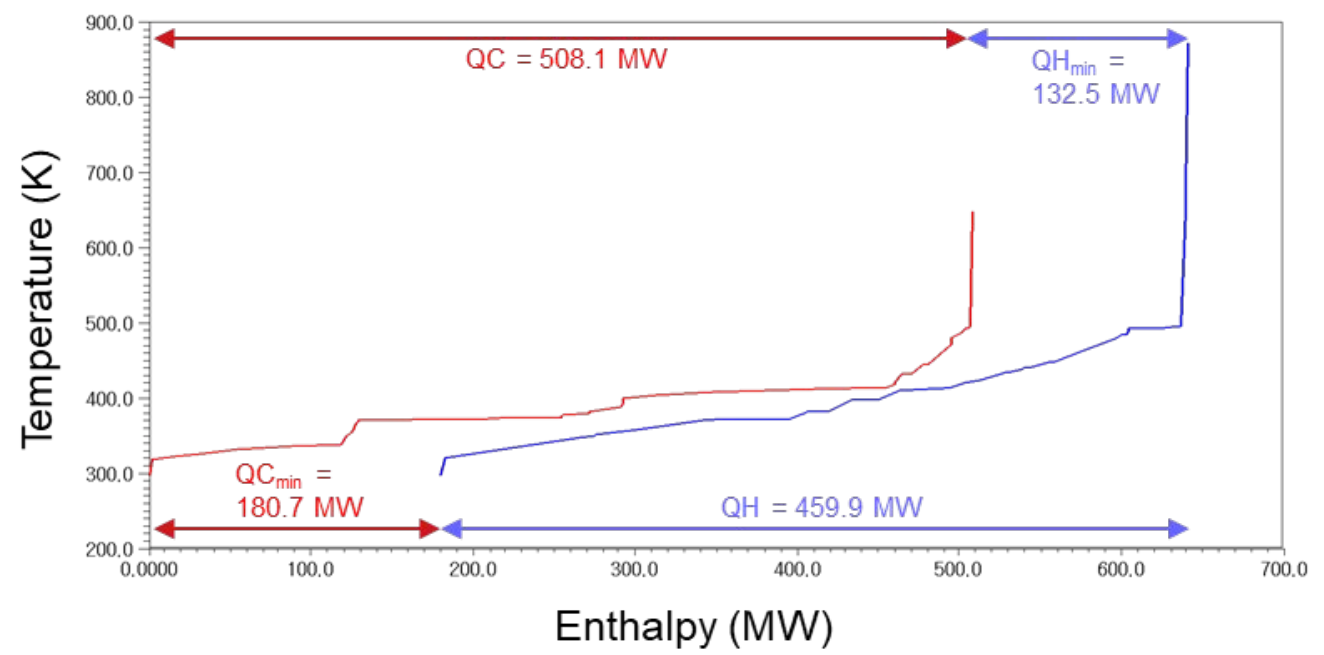

Figure S1. Composite curve. The minimum temperature difference $\left(\Delta \mathrm{T}_{\min }\right)$ is $10 \mathrm{~K} \mathrm{QH}_{\text {min }}$ : total minimum heating utility requirement, $\mathrm{QC}_{\min }$ : total minimum cooling utility requirement. This was obtained after introducing compressor (CP-2) and cooler (C-1). 


\section{Total capital investment}

Table S3. Project cost worksheet.

\begin{tabular}{|c|c|c|c|}
\hline Areas & & & stalled cost \\
\hline Biomass fractionation & & $\$$ & $78,764,842$ \\
\hline 1,5-PDO production & & $\$$ & $18,813,105$ \\
\hline HMF production & & $\$$ & $111,964,049$ \\
\hline FDCA production & & $\$$ & $10,091,713$ \\
\hline FDCA separation & & $\$$ & $6,020,148$ \\
\hline Wastewater treatment & & $\$$ & $24,885,974$ \\
\hline Storage & & $\$$ & $6,876,212$ \\
\hline Heat and power generation & & $\$$ & $83,041,385$ \\
\hline Utilities & & $\$$ & $7,953,071$ \\
\hline Total installed equipment cost & & $\$$ & $348,410,499$ \\
\hline Warehouse & $4.0 \%$ of ISBL ${ }^{*}$ & $\$$ & $9,026,154$ \\
\hline Site development & $9.0 \%$ of ISBL ${ }^{*}$ & $\$$ & $20,308,847$ \\
\hline Additional piping & $4.5 \%$ of ISBL ${ }^{*}$ & $\$$ & $10,154,424$ \\
\hline Total direct cost (TDC) & & $\$$ & $387,899,924$ \\
\hline Prorateable expenses & $10.0 \%$ of TDC & $\$$ & $38,789,992$ \\
\hline Field expenses & $10.0 \%$ of TDC & $\$$ & $38,789,992$ \\
\hline Home office \& construction fee & $20.0 \%$ of TDC & $\$$ & $77,579,985$ \\
\hline Project contingency & $10.0 \%$ of TDC & $\$$ & $38,789,992$ \\
\hline Other costs (start-up, permits, etc.) & $10.0 \%$ of TDC & $\$$ & $38,789,992$ \\
\hline Total indirect cost & & $\$$ & $232,739,954$ \\
\hline Fixed capital investment (FCI) & & $\$$ & $620,639,878$ \\
\hline Land & & $\$$ & $1,848,000$ \\
\hline Working capital & $5.0 \%$ of FCI & $\$$ & $31,031,994$ \\
\hline Total capital investment (TCI) & & $\$$ & $653,519,871$ \\
\hline
\end{tabular}




\section{Discounted cash flow analysis}

Table S4. Discounted cash flow calculations.

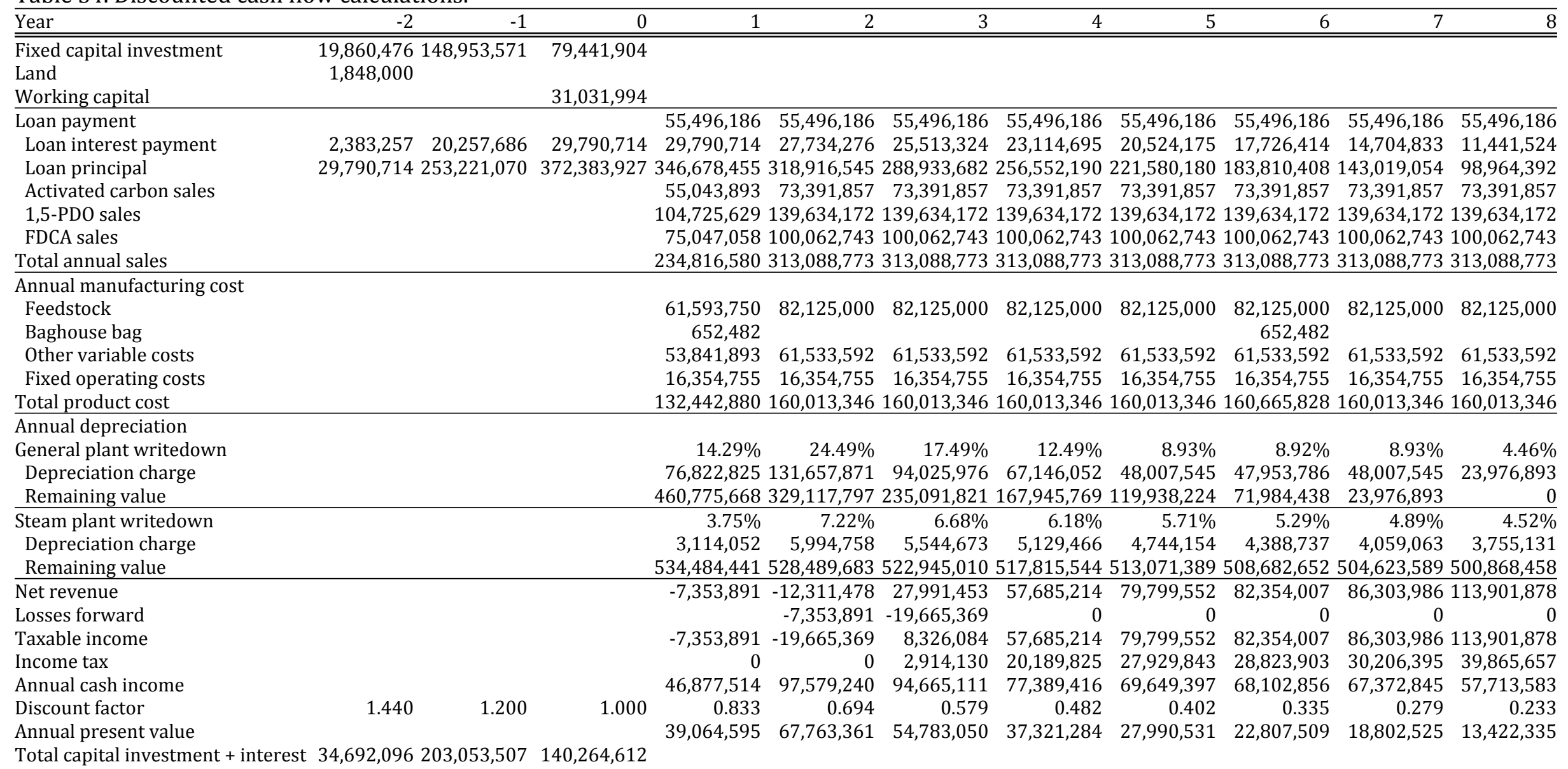

Total capital investment + interest 34,692,096 203,053,507 140,264,612

Net present worth 


\begin{tabular}{|c|c|c|c|c|c|c|c|c|c|c|c|}
\hline Year & 9 & 10 & 11 & 12 & 13 & 14 & 15 & 16 & 17 & 18 & 19 \\
\hline \multicolumn{12}{|l|}{$\begin{array}{l}\text { Fixed capital investment } \\
\text { Land } \\
\text { Working capital }\end{array}$} \\
\hline Loan payment & $55,496,186$ & $55,496,186$ & 0 & 0 & 0 & 0 & 0 & 0 & 0 & 0 & 0 \\
\hline Loan interest payment & $7,917,151$ & $4,110,829$ & 0 & 0 & 0 & 0 & 0 & 0 & 0 & 0 & 0 \\
\hline Loan principal & $51,385,358$ & 0 & 0 & 0 & 0 & 0 & 0 & 0 & 0 & 0 & 0 \\
\hline Activated carbon sales & $73,391,857$ & $73,391,857$ & $73,391,857$ & $73,391,857$ & $7 \quad 73,391,857$ & $73,391,857$ & $73,391,857$ & $73,391,857$ & $73,391,857$ & $73,391,857$ & $73,391,857$ \\
\hline 1,5-PDO sales & $139,634,172$ & $139,634,172$ & $139,634,172$ & $139,634,172$ & $139,634,172$ & $139,634,172$ & $139,634,172$ & $139,634,172$ & $139,634,172$ & $139,634,172$ & $139,634,172$ \\
\hline FDCA sales & $100,062,743$ & $100,062,743$ & $100,062,743$ & $100,062,743$ & $100,062,743$ & $100,062,743$ & $100,062,743$ & $100,062,743$ & $100,062,743$ & $100,062,743$ & $100,062,743$ \\
\hline Total annual sales & $313,088,773$ & $313,088,773$ & $313,088,773$ & $313,088,773$ & $313,088,773$ & $313,088,773$ & $313,088,773$ & $313,088,773$ & $313,088,773$ & $313,088,773$ & $313,088,773$ \\
\hline \multicolumn{12}{|c|}{ Annual manufacturing cost } \\
\hline $\begin{array}{l}\text { Feedstock } \\
\text { Baghouse bag }\end{array}$ & $82,125,000$ & $82,125,000$ & $\begin{array}{r}82,125,000 \\
652,482\end{array}$ & $82,125,000$ & $82,125,000$ & $82,125,000$ & $82,125,000$ & $\begin{array}{r}82,125,000 \\
652,482\end{array}$ & $82,125,000$ & $82,125,000$ & $82,125,000$ \\
\hline Other variable costs & $61,533,592$ & $61,533,592$ & $61,533,592$ & $61,533,592$ & $61,533,592$ & $61,533,592$ & $61,533,592$ & $61,533,592$ & $61,533,592$ & $61,533,592$ & $61,533,592$ \\
\hline Fixed operating costs & $16,354,755$ & $16,354,755$ & $16,354,755$ & $16,354,755$ & $16,354,755$ & $16,354,755$ & $16,354,755$ & $16,354,755$ & $16,354,755$ & $16,354,755$ & $16,354,755$ \\
\hline Total product cost & $160,013,346$ & $160,013,346$ & $160,665,828$ & $160,013,346$ & $160,013,346$ & $160,013,346$ & $160,013,346$ & $160,665,828$ & $160,013,346$ & $160,013,346$ & $160,013,346$ \\
\hline \multicolumn{12}{|l|}{$\begin{array}{l}\text { Annual depreciation } \\
\text { General plant writedown } \\
\text { Depreciation charge } \\
\text { Remaining value } \\
\end{array}$} \\
\hline Steam plant writedown & $4.46 \%$ & $4.46 \%$ & $4.46 \%$ & $4.46 \%$ & $4.46 \%$ & $4.46 \%$ & $4.46 \%$ & $4.46 \%$ & $4.46 \%$ & $4.46 \%$ & $4.46 \%$ \\
\hline Depreciation charge & $3,705,307$ & $3,704,476$ & $3,705,307$ & $3,704,476$ & $3,705,307$ & $3,704,476$ & $3,705,307$ & $3,704,476$ & $3,705,307$ & $3,704,476$ & $3,705,307$ \\
\hline Remaining value & $497,163,151$ & $493,458,675$ & $489,753,368$ & $486,048,892$ & $482,343,586$ & $478,639,109$ & $474,933,803$ & $471,229,327$ & $467,524,020$ & $463,819,544$ & $460,114,237$ \\
\hline Net revenue & $141,452,969$ & $145,260,122$ & $148,717,638$ & $149,370,950$ & $149,370,120$ & $149,370,950$ & $149,370,120$ & $148,718,468$ & $149,370,120$ & $149,370,950$ & $149,370,120$ \\
\hline Losses forward & 0 & 0 & 0 & 0 & 0 & 0 & 0 & 0 & 0 & 0 & 0 \\
\hline Taxable income & $141,452,969$ & $145,260,122$ & $148,717,638$ & $149,370,950$ & $149,370,120$ & $149,370,950$ & $149,370,120$ & $148,718,468$ & $149,370,120$ & $149,370,950$ & $149,370,120$ \\
\hline Income tax & $49,508,539$ & $50,841,043$ & $52,051,173$ & $52,279,833$ & $52,279,542$ & $52,279,833$ & $52,279,542$ & $52,051,464$ & $52,279,542$ & $52,279,833$ & $52,279,542$ \\
\hline Annual cash income & $48,070,701$ & $46,738,198$ & $100,371,771$ & $100,795,594$ & $100,795,885$ & $100,795,594$ & $100,795,885$ & $100,371,480$ & $100,795,885$ & $100,795,594$ & 100,795,885 \\
\hline Discount factor & 0.194 & 0.162 & 0.135 & 0.112 & 0.093 & 0.078 & 0.065 & 0.054 & 0.045 & 0.038 & 0.031 \\
\hline $\begin{array}{l}\text { Annual present value } \\
\text { Total capital investment } \\
\text { Net present worth }\end{array}$ & $9,316,424$ & $7,548,480$ & $13,508,835$ & $11,304,897$ & $9,420,774$ & $7,850,623$ & $6,542,204$ & $5,428,882$ & $4,543,198$ & $3,785,987$ & $3,154,998$ \\
\hline
\end{tabular}




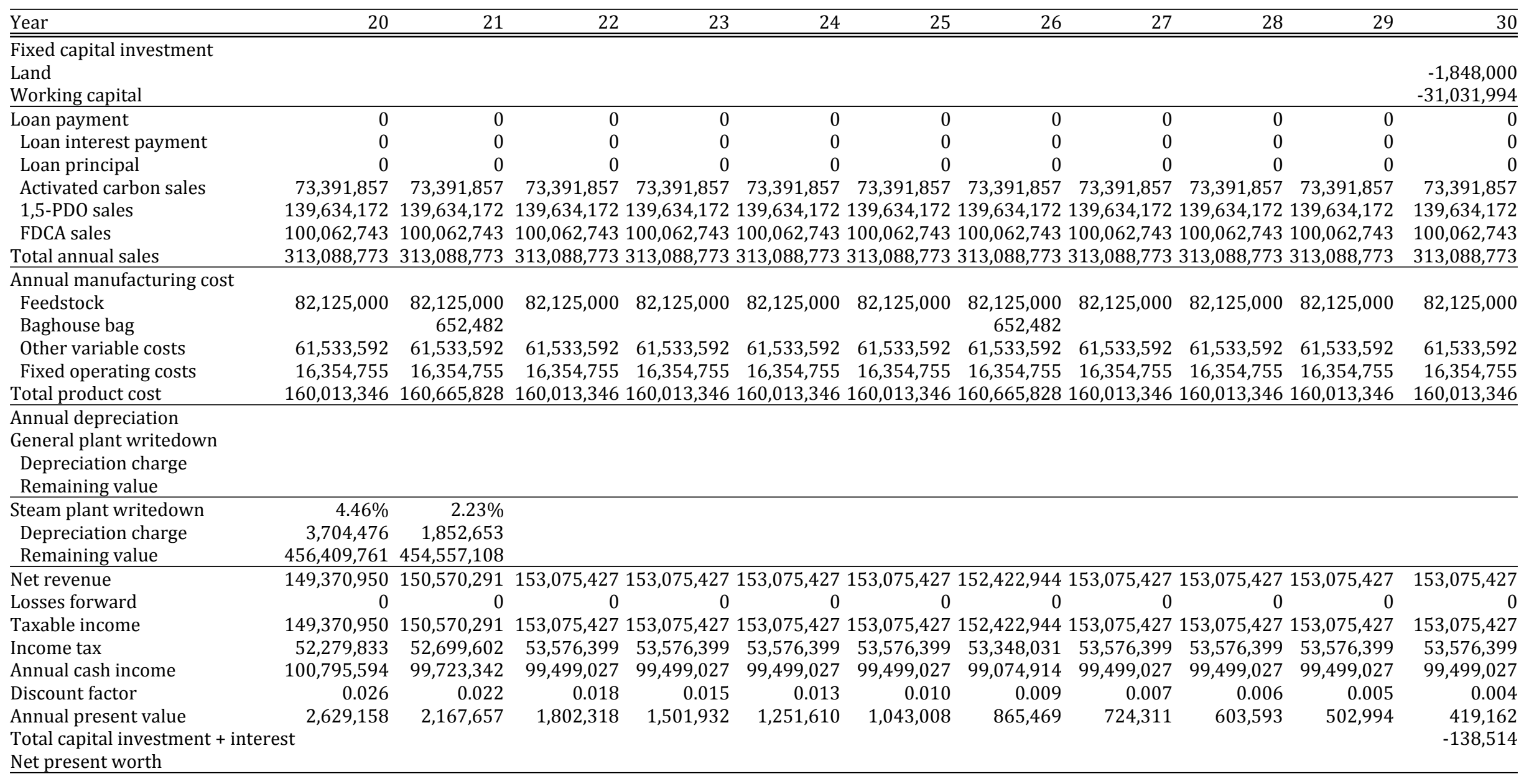




\section{LCA data and results}

Table S5. LCA data sets.

\begin{tabular}{|c|c|c|}
\hline Materials & Name of data set & Database \\
\hline Acetic acid & cetic acid, without water, in $98 \%$ solution state $\{\mathrm{GLO}\} \mid$ market for | Cut-off, $\mathrm{U}$ & Ecoinvent 3.6 \\
\hline Ash & Dummy_Disposal, fly ash, to unspecified landfill/kg/RNA & Ecoinvent 3.6 \\
\hline $\begin{array}{l}\text { Average incineration } \\
\text { residue }\end{array}$ & Average incineration residue $\{$ RoW $\} \mid$ market for | Cut-off, $U$ & Ecoinvent 3.6 \\
\hline Biomass & Wood chips, wet, measured as dry mass $\{$ RoW $\} \mid$ market for | Cut-off, $U$ & Ecoinvent 3.6 \\
\hline Chemical factory & Chemical factory, organics $\{\mathrm{GLO}\} \mid$ market for $\mid$ Cut-off, $\mathrm{U}$ & Ecoinvent 3.6 \\
\hline $\mathrm{CO}_{2}$ & Carbon dioxide & Ecoinvent 3.6 \\
\hline Electricity & Electricity, high voltage $\{\mathrm{GLO}\} \mid$ market for | Cut-off, $\mathrm{U}$ & Ecoinvent 3.6 \\
\hline Electricity & Electricity, medium voltage $\{$ RoW $\}$ | electricity production, hard coal | Cut-off, $U$ & Ecoinvent 3.6 \\
\hline Electricity & Electricity, medium voltage $\{\mathrm{RER}\} \mid$ market for | Cut-off, $\mathrm{U}$ & Ecoinvent 3.6 \\
\hline Electricity & $\begin{array}{l}\text { Electricity, high voltage }\{\text { RoW }\} \text { | electricity production, hydro, run-of-river | } \\
\text { Cut-off, } U\end{array}$ & Ecoinvent 3.6 \\
\hline Electricity & $\begin{array}{l}\text { Electricity, high voltage }\{\mathrm{RoW}\} \text { | electricity production, nuclear, pressure water } \\
\text { reactor | Cut-off, U }\end{array}$ & Ecoinvent 3.6 \\
\hline Electricity & $\begin{array}{l}\text { Electricity, high voltage }\{\text { RoW }\} \text { | electricity production, wind, >3MW turbine, } \\
\text { onshore | Cut-off, U }\end{array}$ & Ecoinvent 3.6 \\
\hline Electricity & $\begin{array}{l}\text { Electricity, high voltage }\{\text { RoW }\} \text { | heat and power co-generation, biogas, gas } \\
\text { engine | Cut-off, } U\end{array}$ & Ecoinvent 3.6 \\
\hline Electricity & $\begin{array}{l}\text { Electricity, high voltage }\{\text { RoW }\} \text { | heat and power co-generation, wood chips, } \\
6667 \mathrm{~kW} \mid \text { Cut-off, } U\end{array}$ & Ecoinvent 3.6 \\
\hline Electricity & $\begin{array}{l}\text { Electricity, high voltage }\{\mathrm{RoW}\} \text { | treatment of blast furnace gas, in power plant | } \\
\text { Cut-off, } U\end{array}$ & Ecoinvent 3.6 \\
\hline Electricity & $\begin{array}{l}\text { Electricity, high voltage }\{\mathrm{RoW}\} \mid \text { treatment of coal gas, in power plant | Cut-off, } \\
\mathrm{U}\end{array}$ & Ecoinvent 3.6 \\
\hline Gypsum & Waste gypsum $\{$ RoW $\}$ | market for waste gypsum| APOS, S & Ecoir \\
\hline $\mathrm{H}_{2}$ & Hydr & Ecoir \\
\hline $\mathrm{H}_{2} \mathrm{O}$ & Water $/ \mathrm{m}^{3}$ & Ecoinvent 3.6 \\
\hline $\mathrm{H}_{2} \mathrm{O}$ & Water, cooling, uns & Ecoinvent 3.6 \\
\hline $\mathrm{H}_{2} \mathrm{O}$ & $\begin{array}{l}\text { Water, completely softened, from decarbonized water, at user }\{\mathrm{GLO}\} \mid \text { market } \\
\text { for | Cut-off, U }\end{array}$ & Ecoinvent 3.6 \\
\hline Hazardous & Hazardous waste, for underground deposit $\{\mathrm{GLO}\} \mid$ market for | Cut-off, $\mathrm{U}$ & Eco \\
\hline Hydrocarbons & Hydr & th 3.6 \\
\hline Heavy oil & oW\} | marke & Ecoinvent 3.6 \\
\hline Heat & rict or industrial, natural gas $\{\mathrm{G}$ & Ecoinvent 3.6 \\
\hline Heat & Heat, district or industrial, natural gas $\{\mathrm{RER}\} \mid$ market group for | Cut-off, $\mathrm{U}$ & Ecoinvent 3.6 \\
\hline Heat & $\begin{array}{l}\text { Heat, district or industrial, other than natural gas }\{R E R\} \mid \text { market group for | } \\
\text { Cut-off, } U\end{array}$ & Ecoinvent 3.6 \\
\hline Lime & | market for | Cut-off, $U$ & \\
\hline $\mathrm{N}_{2}$ & Nitro & t 3.6 \\
\hline $\mathrm{NaCl}$ & Sodil & Ecoinvent 3.6 \\
\hline $\mathrm{NaOH}$ & $\begin{array}{l}\text { Sodium hydroxide, without water, in } 50 \% \text { solution state }\{\text { GLO }\} \text { | market for | } \\
\text { Cut-off, } U\end{array}$ & Ecoinvent 3.6 \\
\hline NMVOC & NMVOC, non-methane volatile organic compounds, unspecified origin & Eco \\
\hline Particulates & $2.5 \mathrm{um}$ & Ecoinvent 3.6 \\
\hline Particulates & $>10$ um & Ecoinvent 3.6 \\
\hline Particulates & Parti & Ecoinvent 3.6 \\
\hline $\mathrm{O}_{2}$ & id $\{$ RoW $\} \mid$ market for $\mid$ Cut & Ecoinvent 3.6 \\
\hline Steam & in chemical industry $\{\mathrm{GLO}\} \mid$ market for | Cut-off, $\mathrm{U}$ & Ecoinvent 3.6 \\
\hline & or | Cut-off, U & Ecoinvent 3.6 \\
\hline Tetrahydrofuran & Tetrahydrofuran $\{$ GLO $\}$ | market for | Cut-off, $U$ & Ecoinvent 3.6 \\
\hline Xylene & Xylene $\{$ GLO $\} \mid$ market for $\mid$ Cut-off, $U$ & Ecoinvent 3.6 \\
\hline
\end{tabular}




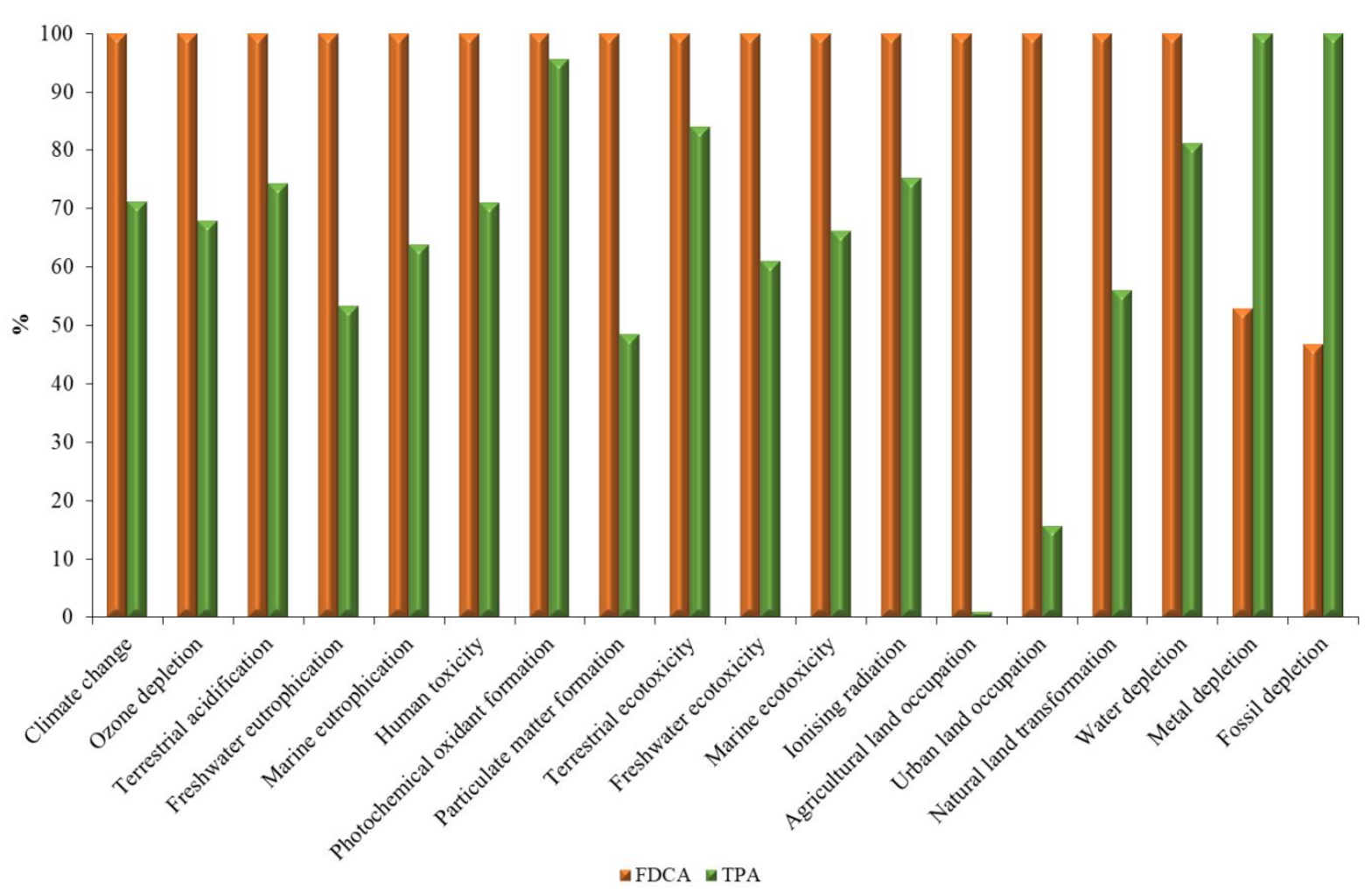

Figure S2. Comparison of environmental impact results for the productions of petroleum-derived TPA and biomass-derived FDCA.

Table S6. Environmental impact results for the two processes.

\begin{tabular}{llll}
\hline & \multicolumn{1}{c}{ Unit } & \multicolumn{1}{c}{ FDCA } & \multicolumn{1}{c}{ TPA } \\
\hline Climate change & $\mathrm{kg} \mathrm{CO}_{2}$ eq & 2.525097 & 1.7996478 \\
Ozone depletion & $\mathrm{kg} \mathrm{CFC}-11$ eq & $1.15 \mathrm{E}-07$ & $7.80 \mathrm{E}-08$ \\
Terrestrial acidification & $\mathrm{kg} \mathrm{SO}_{2}$ eq & 0.008171797 & 0.006078061 \\
Freshwater eutrophication & $\mathrm{kg} \mathrm{P} \mathrm{eq}$ & 0.000783894 & 0.000418913 \\
Marine eutrophication & $\mathrm{kg} \mathrm{N} \mathrm{eq}$ & 0.000342371 & 0.00021877 \\
Human toxicity & $\mathrm{kg} \mathrm{1,4-DB} \mathrm{eq}$ & 0.61190343 & 0.43471187 \\
Photochemical oxidant formation & $\mathrm{kg} \mathrm{NMVOC}$ & 0.005297111 & 0.00506546 \\
Particulate matter formation & $\mathrm{kg} \mathrm{PM} 10 \mathrm{eq}$ & 0.004561103 & 0.002213083 \\
Terrestrial ecotoxicity & $\mathrm{kg} \mathrm{1,4-DB} \mathrm{eq}$ & 0.000134273 & 0.000112814 \\
Freshwater ecotoxicity & $\mathrm{kg} \mathrm{1,4-DB} \mathrm{eq}$ & 0.020566024 & 0.012544208 \\
Marine ecotoxicity & $\mathrm{kg} \mathrm{1,4-DB} \mathrm{eq}$ & 0.018866095 & 0.012498356 \\
Ionising radiation & $\mathrm{kBq} \mathrm{U235} \mathrm{eq}$ & 0.19295201 & 0.1451461 \\
Agricultural land occupation & $\mathrm{m}^{2} \mathrm{a}$ & 7.8894258 & 0.075566638 \\
Urban land occupation & $\mathrm{m}^{2} \mathrm{a}$ & 0.047759697 & 0.007445328 \\
Natural land transformation & $\mathrm{m}^{2}$ & 0.000249912 & 0.000139941 \\
Water depletion & $\mathrm{m}^{3}$ & 0.032934216 & 0.026776463 \\
Metal depletion & $\mathrm{kg} \mathrm{Fe} \mathrm{eq}$ & 0.032031813 & 0.060617777 \\
Fossil depletion & $\mathrm{kg} \mathrm{oil} \mathrm{eq}$ & 0.54646789 & 1.1672443 \\
\hline
\end{tabular}




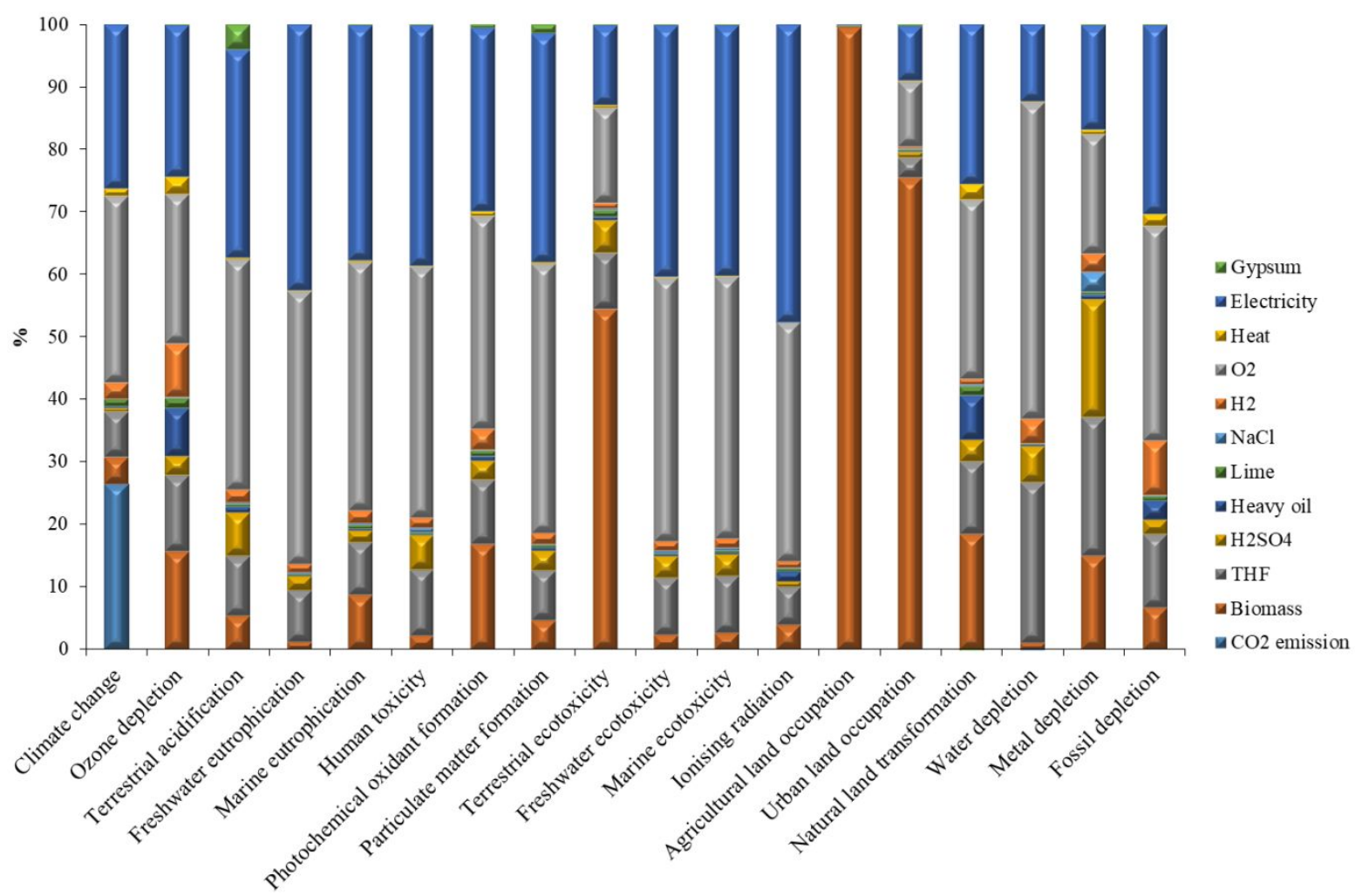

Figure S3. Comparison of environmental impact for input and output sources of biomass-derived FDCA production. 
Table S7. Environmental impact results for biomass-derived FDCA production.

\begin{tabular}{|c|c|c|c|c|c|c|c|c|c|c|c|c|c|}
\hline & Unit & $\mathrm{CO}_{2}$ emission & Biomass & THF & $\mathrm{H}_{2} \mathrm{SO}_{4}$ & Heavy oil & $\mathrm{Ca}(\mathrm{OH})_{2}$ & $\mathrm{NaCl}$ & $\mathrm{H}_{2}$ & $\mathrm{O}_{2}$ & Heat & Electricity & Gypsum \\
\hline Climate change & $\begin{array}{l}\mathrm{kg} \mathrm{CO}_{2} \\
\mathrm{eq}\end{array}$ & 0.6690 & 0.1078 & 0.1838 & 0.0146 & 0.0063 & 0.0298 & 0.0028 & 0.0658 & 0.7540 & 0.0272 & 0.6632 & 0.0008 \\
\hline $\begin{array}{l}\text { Ozone } \\
\text { depletion }\end{array}$ & $\begin{array}{l}\text { kg CFC- } \\
11 \text { eq }\end{array}$ & & $\begin{array}{r}0.000000018 \\
0\end{array}$ & $\begin{array}{r}0.000000014 \\
1\end{array}$ & $\begin{array}{r}0.000000003 \\
4\end{array}$ & $\begin{array}{r}0.000000008 \\
9\end{array}$ & $\begin{array}{r}0.000000001 \\
8\end{array}$ & $\begin{array}{r}0.000000000 \\
2\end{array}$ & $\begin{array}{r}0.000000009 \\
9\end{array}$ & 0.000000027 & $\begin{array}{r}0.000000003 \\
2\end{array}$ & $\begin{array}{r}0.000000027 \\
8\end{array}$ & $\begin{array}{r}0.000000000 \\
2\end{array}$ \\
\hline $\begin{array}{l}\text { Terrestrial } \\
\text { acidification }\end{array}$ & $\begin{array}{l}\mathrm{kg} \mathrm{SO} \\
\text { eq }\end{array}$ & & 0.0004 & 0.0008 & 0.0006 & 0.0001 & 0.0000 & 0.0000 & 0.0002 & 0.0030 & 0.0000 & 0.0027 & 0.0003 \\
\hline $\begin{array}{l}\text { Freshwater } \\
\text { eutrophication }\end{array}$ & $\mathrm{kg} P$ eq & & 0.0000 & 0.0001 & 0.0000 & 0.0000 & 0.0000 & 0.0000 & 0.0000 & 0.0003 & 0.0000 & 0.0003 & 0.0000 \\
\hline $\begin{array}{l}\text { Marine } \\
\text { eutrophication }\end{array}$ & kg N eq & & 0.0000 & 0.0000 & 0.0000 & 0.0000 & 0.0000 & 0.0000 & 0.0000 & 0.0001 & 0.0000 & 0.0001 & 0.0000 \\
\hline Human toxicity & $\begin{array}{l}\text { kg 1,4- } \\
\text { DB eq }\end{array}$ & & 0.0142 & 0.0641 & 0.0336 & 0.0011 & 0.0016 & 0.0043 & 0.0099 & 0.2466 & 0.0007 & 0.2351 & 0.0006 \\
\hline $\begin{array}{l}\text { Photochemical } \\
\text { oxidant } \\
\text { formation }\end{array}$ & $\begin{array}{l}\mathrm{kg} \\
\text { NMVOC }\end{array}$ & & 0.0009 & 0.0005 & 0.0002 & 0.0000 & 0.0000 & 0.0000 & 0.0002 & 0.0018 & 0.0000 & 0.0016 & 0.0000 \\
\hline $\begin{array}{l}\text { Particulate } \\
\text { matter } \\
\text { formation }\end{array}$ & $\begin{array}{l}\mathrm{kg} \\
\text { PM10 } \\
\text { eq }\end{array}$ & & 0.0002 & 0.0004 & 0.0001 & 0.0000 & 0.0000 & 0.0000 & 0.0001 & 0.0020 & 0.0000 & 0.0017 & 0.0001 \\
\hline $\begin{array}{l}\text { Terrestrial } \\
\text { ecotoxicity }\end{array}$ & $\begin{array}{l}\operatorname{kg} 1,4- \\
\text { DB eq }\end{array}$ & & 0.0001 & 0.0000 & 0.0000 & 0.0000 & 0.0000 & 0.0000 & 0.0000 & 0.0000 & 0.0000 & 0.0000 & 0.0000 \\
\hline $\begin{array}{l}\text { Freshwater } \\
\text { ecotoxicity }\end{array}$ & $\begin{array}{l}\operatorname{kg} 1,4- \\
\text { DB eq }\end{array}$ & & 0.0005 & 0.0019 & 0.0007 & 0.0000 & 0.0000 & 0.0001 & 0.0003 & 0.0087 & 0.0000 & 0.0083 & 0.0000 \\
\hline $\begin{array}{l}\text { Marine } \\
\text { ecotoxicity }\end{array}$ & $\begin{array}{l}\operatorname{kg} 1,4- \\
\text { DB eq }\end{array}$ & & 0.0005 & 0.0017 & 0.0007 & 0.0000 & 0.0001 & 0.0001 & 0.0003 & 0.0079 & 0.0000 & 0.0075 & 0.0000 \\
\hline $\begin{array}{l}\text { Ionising } \\
\text { radiation }\end{array}$ & $\begin{array}{l}\mathrm{kBq} \\
\mathrm{U} 235 \mathrm{eq}\end{array}$ & & 0.0076 & 0.0117 & 0.0018 & 0.0032 & 0.0009 & 0.0003 & 0.0019 & 0.0738 & 0.0002 & 0.0916 & 0.0001 \\
\hline $\begin{array}{l}\text { Agricultural } \\
\text { land occupation }\end{array}$ & $\mathrm{m}^{2} \mathrm{a}$ & & 7.8514 & 0.0091 & 0.0013 & 0.0001 & 0.0014 & 0.0004 & 0.0006 & 0.0102 & 0.0000 & 0.0148 & 0.0001 \\
\hline $\begin{array}{l}\text { Urban land } \\
\text { occupation }\end{array}$ & $\mathrm{m}^{2} \mathrm{a}$ & & 0.0361 & 0.0015 & 0.0005 & 0.0001 & 0.0001 & 0.0001 & 0.0002 & 0.0050 & 0.0000 & 0.0042 & 0.0001 \\
\hline $\begin{array}{l}\text { Natural land } \\
\text { transformation }\end{array}$ & $\mathrm{m}^{2}$ & & 0.0000 & 0.0000 & 0.0000 & 0.0000 & 0.0000 & 0.0000 & 0.0000 & 0.0001 & 0.0000 & 0.0001 & -0.0000 \\
\hline Water depletion & $\mathrm{m}^{3}$ & & 0.0004 & 0.0086 & 0.0019 & 0.0001 & 0.0000 & -0.0000 & 0.0013 & 0.0169 & 0.0000 & 0.0041 & 0.0000 \\
\hline Metal depletion & $\mathrm{kg} \mathrm{Fe} \mathrm{eq}$ & & 0.0048 & 0.0071 & 0.0061 & 0.0002 & 0.0002 & 0.0010 & 0.0010 & 0.0061 & 0.0002 & 0.0054 & 0.0000 \\
\hline Fossil depletion & kg oil eq & & 0.0365 & 0.0641 & 0.0134 & 0.0167 & 0.0037 & 0.0007 & 0.0479 & 0.1873 & 0.0104 & 0.1653 & 0.0004 \\
\hline
\end{tabular}




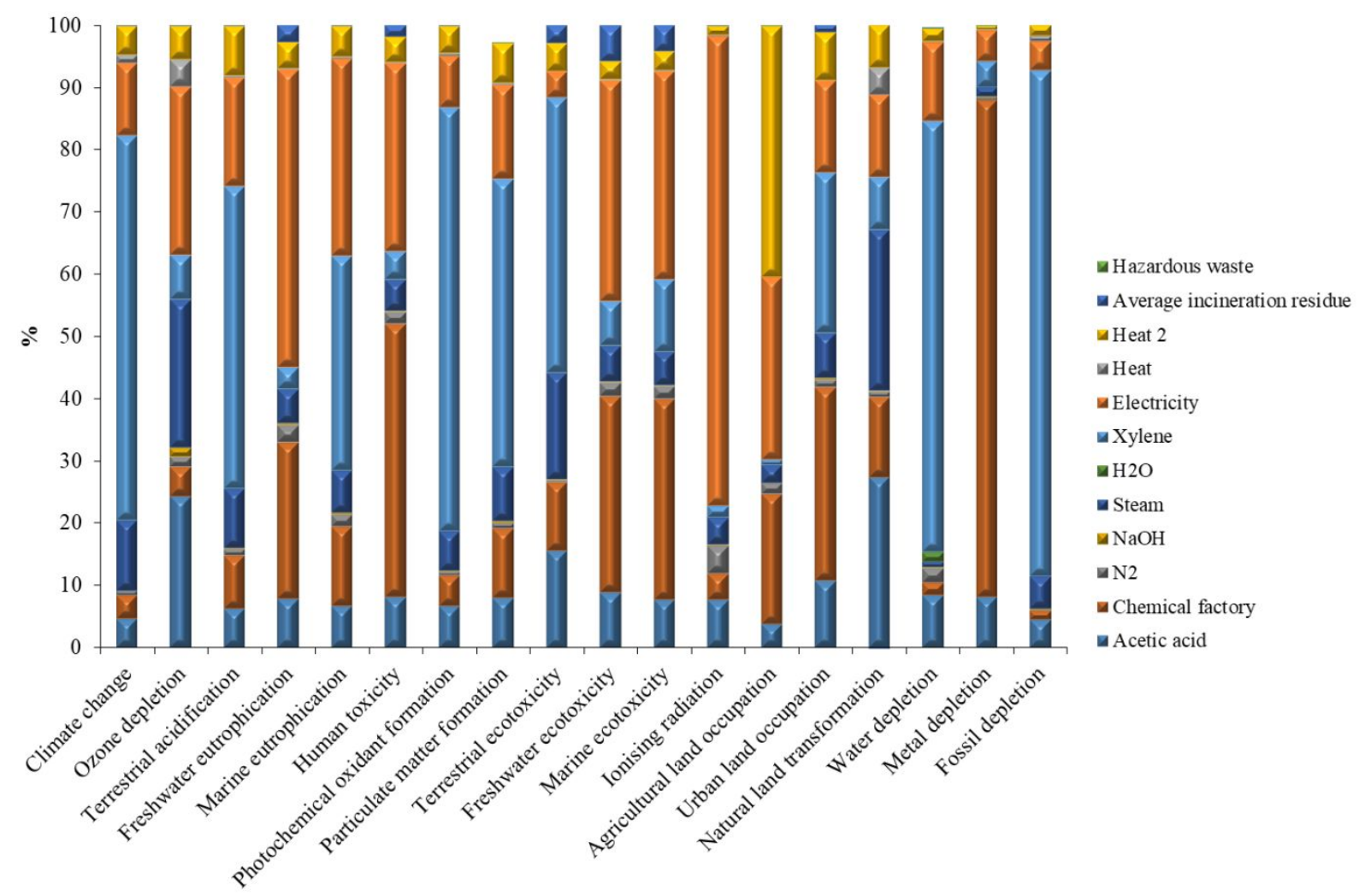

Figure S4. Comparison of environmental impact for input and output sources of petroleumderived TPA production. 
Table S8. Environmental impact results for petroleum-derived TPA production.

\begin{tabular}{|c|c|c|c|c|c|c|c|c|c|c|c|c|}
\hline & Unit & Acetic acid & $\begin{array}{c}\text { Chemical } \\
\text { factory }\end{array}$ & $\mathrm{N}_{2}$ & $\mathrm{NaOH}$ & Steam & $\mathrm{H}_{2} \mathrm{O}$ & Xylene & Electricity & Heat & Residue & Waste \\
\hline Climate change & $\mathrm{kg} \mathrm{CO}_{2}$ eq & 0.0859 & 0.0665 & 0.0124 & 0.0020 & 0.2023 & 0.0000 & 1.1125 & 0.2079 & 0.1078 & 0.0022 & 0.0001 \\
\hline Ozone depletion & kg CFC-11 eq & 0.0000 & 0.0000 & 0.0000 & 0.0000 & 0.0000 & 0.0000 & 0.0000 & 0.0000 & 0.0000 & 0.0000 & 0.0000 \\
\hline Terrestrial acidification & $\mathrm{kg} \mathrm{SO} \mathrm{eq}_{2}$ & 0.0004 & 0.0005 & 0.0001 & 0.0000 & 0.0006 & 0.0000 & 0.0030 & 0.0011 & 0.0005 & 0.0000 & 0.0000 \\
\hline Freshwater eutrophication & $\mathrm{kg} P$ eq & 0.0000 & 0.0001 & 0.0000 & 0.0000 & 0.0000 & 0.0000 & 0.0000 & 0.0002 & 0.0000 & 0.0000 & 0.0000 \\
\hline Marine eutrophication & $\operatorname{kg~N}$ eq & 0.0000 & 0.0000 & 0.0000 & 0.0000 & 0.0000 & 0.0000 & 0.0001 & 0.0001 & 0.0000 & 0.0000 & 0.0000 \\
\hline Human toxicity & $\operatorname{kg} 1,4$-DB eq & 0.0356 & 0.1910 & 0.0078 & 0.0011 & 0.0217 & 0.0000 & 0.0197 & 0.1314 & 0.0181 & 0.0083 & 0.0000 \\
\hline Photochemical oxidant formation & kg NMVOC & 0.0003 & 0.0003 & 0.0000 & 0.0000 & 0.0003 & 0.0000 & 0.0034 & 0.0004 & 0.0002 & 0.0000 & 0.0000 \\
\hline Particulate matter formation & kg PM10 eq & 0.0002 & 0.0002 & 0.0000 & 0.0000 & 0.0002 & 0.0000 & 0.0010 & 0.0003 & 0.0001 & 0.0000 & 0.0000 \\
\hline Terrestrial ecotoxicity & $\operatorname{kg} 1,4-\mathrm{DB}$ eq & 0.0000 & 0.0000 & 0.0000 & 0.0000 & 0.0000 & 0.0000 & 0.0000 & 0.0000 & 0.0000 & 0.0000 & 0.0000 \\
\hline Freshwater ecotoxicity & kg 1,4-DB eq & 0.0011 & 0.0040 & 0.0003 & 0.0000 & 0.0007 & 0.0000 & 0.0009 & 0.0045 & 0.0004 & 0.0007 & 0.0000 \\
\hline Marine ecotoxicity & $\operatorname{kg} 1,4$-DB eq & 0.0010 & 0.0040 & 0.0002 & 0.0000 & 0.0007 & 0.0000 & 0.0015 & 0.0042 & 0.0004 & 0.0005 & 0.0000 \\
\hline Ionising radiation & $\mathrm{kBq}$ U235 eq & 0.0112 & 0.0063 & 0.0064 & 0.0003 & 0.0062 & 0.0000 & 0.0029 & 0.1094 & 0.0024 & 0.0001 & 0.0000 \\
\hline Agricultural land occupation & $m^{2} a$ & 0.0029 & 0.0158 & 0.0013 & 0.0001 & 0.0022 & 0.0000 & 0.0006 & 0.0222 & 0.0304 & 0.0000 & 0.0000 \\
\hline Urban land occupation & $\mathrm{m}^{2} \mathrm{a}$ & 0.0008 & 0.0023 & 0.0001 & 0.0000 & 0.0005 & 0.0000 & 0.0019 & 0.0011 & 0.0006 & 0.0001 & 0.0000 \\
\hline Natural land transformation & $\mathrm{m}^{2}$ & 0.0000 & 0.0000 & 0.0000 & 0.0000 & 0.0000 & 0.0000 & 0.0000 & 0.0000 & 0.0000 & -0.0000 & 0.0000 \\
\hline Water depletion & $\mathrm{m}^{3}$ & 0.0023 & 0.0006 & 0.0006 & 0.0001 & 0.0002 & 0.0004 & 0.0186 & 0.0034 & 0.0006 & 0.0000 & 0.0000 \\
\hline Metal depletion & $\mathrm{kg} \mathrm{Fe} \mathrm{eq}$ & 0.0049 & 0.0484 & 0.0002 & 0.0001 & 0.0009 & 0.0000 & 0.0025 & 0.0029 & 0.0005 & 0.0000 & 0.0000 \\
\hline Fossil depletion & kg oil eq & 0.0537 & 0.0159 & 0.0033 & 0.0005 & 0.0614 & 0.0000 & 0.9475 & 0.0544 & 0.0302 & 0.0003 & 0.0000 \\
\hline
\end{tabular}


Table S9. Climate change results for biomass-derived FDCA production [kg $\left.\mathrm{CO}_{2} \mathrm{eq}\right]$.

\begin{tabular}{|c|c|c|c|c|c|c|c|c|c|c|c|c|}
\hline FDCA & $\begin{array}{c}\mathrm{CO}_{2} \\
\text { emission } \\
\end{array}$ & Biomass & THF & $\mathrm{H}_{2} \mathrm{SO}_{4}$ & Oil & $\mathrm{Ca}(\mathrm{OH})_{2}$ & $\mathrm{NaCl}$ & $\mathrm{H}_{2}$ & $\mathrm{O}_{2}$ & Gypsum & Heat & Electricity \\
\hline $\begin{array}{l}\text { Wood } \\
\text { chips }\end{array}$ & 0.669036 & 0.108 & 0.184 & 0.015 & 0.006 & 0.030 & 0.003 & 0.066 & 0.754 & 0.001 & 0.027 & 0.054 \\
\hline Wind & 0.669036 & 0.108 & 0.184 & 0.015 & 0.006 & 0.030 & 0.003 & 0.066 & 0.754 & 0.001 & 0.027 & 0.028 \\
\hline Nuclear & 0.669036 & 0.108 & 0.184 & 0.015 & 0.006 & 0.030 & 0.003 & 0.066 & 0.754 & 0.001 & 0.027 & 0.010 \\
\hline Hydro & 0.669036 & 0.108 & 0.184 & 0.015 & 0.006 & 0.030 & 0.003 & 0.066 & 0.754 & 0.001 & 0.027 & 0.004 \\
\hline $\begin{array}{l}\text { Hard } \\
\text { coal }\end{array}$ & 0.669036 & 0.108 & 0.184 & 0.015 & 0.006 & 0.030 & 0.003 & 0.066 & 0.754 & 0.001 & 0.027 & 0.898 \\
\hline Coal gas & 0.669036 & 0.108 & 0.184 & 0.015 & 0.006 & 0.030 & 0.003 & 0.066 & 0.754 & 0.001 & 0.027 & 1.142 \\
\hline Biogas & 0.669036 & 0.108 & 0.184 & 0.015 & 0.006 & 0.030 & 0.003 & 0.066 & 0.754 & 0.001 & 0.027 & 0.129 \\
\hline $\begin{array}{l}\text { Blast } \\
\text { furnace } \\
\text { gas }\end{array}$ & 0.669036 & 0.108 & 0.184 & 0.015 & 0.006 & 0.030 & 0.003 & 0.066 & 0.754 & 0.001 & 0.027 & 1.673 \\
\hline $\begin{array}{l}\text { Natural } \\
\text { gas }\end{array}$ & 0.669036 & 0.108 & 0.184 & 0.015 & 0.006 & 0.030 & 0.003 & 0.066 & 0.754 & 0.001 & 0.027 & 0.663 \\
\hline TPA & & $\begin{array}{c}\text { Acetic } \\
\text { acid }\end{array}$ & $\begin{array}{l}\text { Chemical } \\
\text { factory }\end{array}$ & Residue & $\mathrm{NaOH}$ & Steam & $\mathrm{H}_{2} \mathrm{O}$ & Xylene & $\mathrm{N}_{2}$ & Waste & Heat & Electricity \\
\hline $\begin{array}{l}\text { Natural } \\
\text { gas }\end{array}$ & & 0.086 & 0.067 & 0.012 & 0.002 & 0.202 & 0.000 & 1.113 & 002 & 0.000 & 0.108 & 0.208 \\
\hline
\end{tabular}

Table S10. Fossil depletion results for biomass-derived FDCA production [kg oil eq].

\begin{tabular}{|c|c|c|c|c|c|c|c|c|c|c|c|}
\hline FDCA & Biomass & THF & $\mathrm{H}_{2} \mathrm{SO}_{4}$ & Heavy oil & $\mathrm{Ca}(\mathrm{OH})_{2}$ & $\mathrm{NaCl}$ & $\mathrm{H}_{2}$ & $\mathrm{O}_{2}$ & Gypsum & Heat & Electricity \\
\hline Wood chips & 0.037 & 0.064 & 0.013 & 0.017 & 0.004 & 0.001 & 0.048 & 0.187 & 0.000 & 0.010 & 0.016 \\
\hline Wind & 0.037 & 0.064 & 0.013 & 0.017 & 0.004 & 0.001 & 0.048 & 0.187 & 0.000 & 0.010 & 0.007 \\
\hline & 0. & 0.064 & 0.013 & 0.017 & 0.004 & 0.00 & 0.048 & 0.187 & 0.000 & 0.010 & 0.003 \\
\hline & 7 & 4 & .013 & & 0 & & 3 & 0.187 & & 0 & 0.001 \\
\hline & & & & & & & & 37 & & 0 & 0.225 \\
\hline Coal gas & 0.037 & 0.064 & 0.013 & 017 & 0.004 & 0.0 & 0.048 & 0.187 & 0.0 & 0.010 & 0.537 \\
\hline Biog & . & 54 & 0 & & 0 & & 0.0 & 0.187 & 0.0 & 0 & 0.009 \\
\hline inace & 7 & 64 & 3 & 7 & 4 & & 0.048 & 0.187 & & 0 & 0.001 \\
\hline al gas & 0.037 & 0.064 & 0.013 & 0.017 & 0.004 & 0.001 & 0.048 & 0.187 & 0.000 & 0.010 & 0.165 \\
\hline TPA & $\begin{array}{c}\text { Acetic } \\
\text { acid }\end{array}$ & $\begin{array}{l}\text { Chemical } \\
\text { factory }\end{array}$ & esidue & $\mathrm{NaOH}$ & eam & ${ }_{2} \mathrm{O}$ & le & $\mathrm{N}_{2}$ & Waste & Ieá & ty \\
\hline Natural gas & 0.054 & 0.016 & 0.003 & 0.001 & 0.061 & 0.000 & 0.948 & 0.000 & 0.000 & 0.030 & 0.054 \\
\hline
\end{tabular}




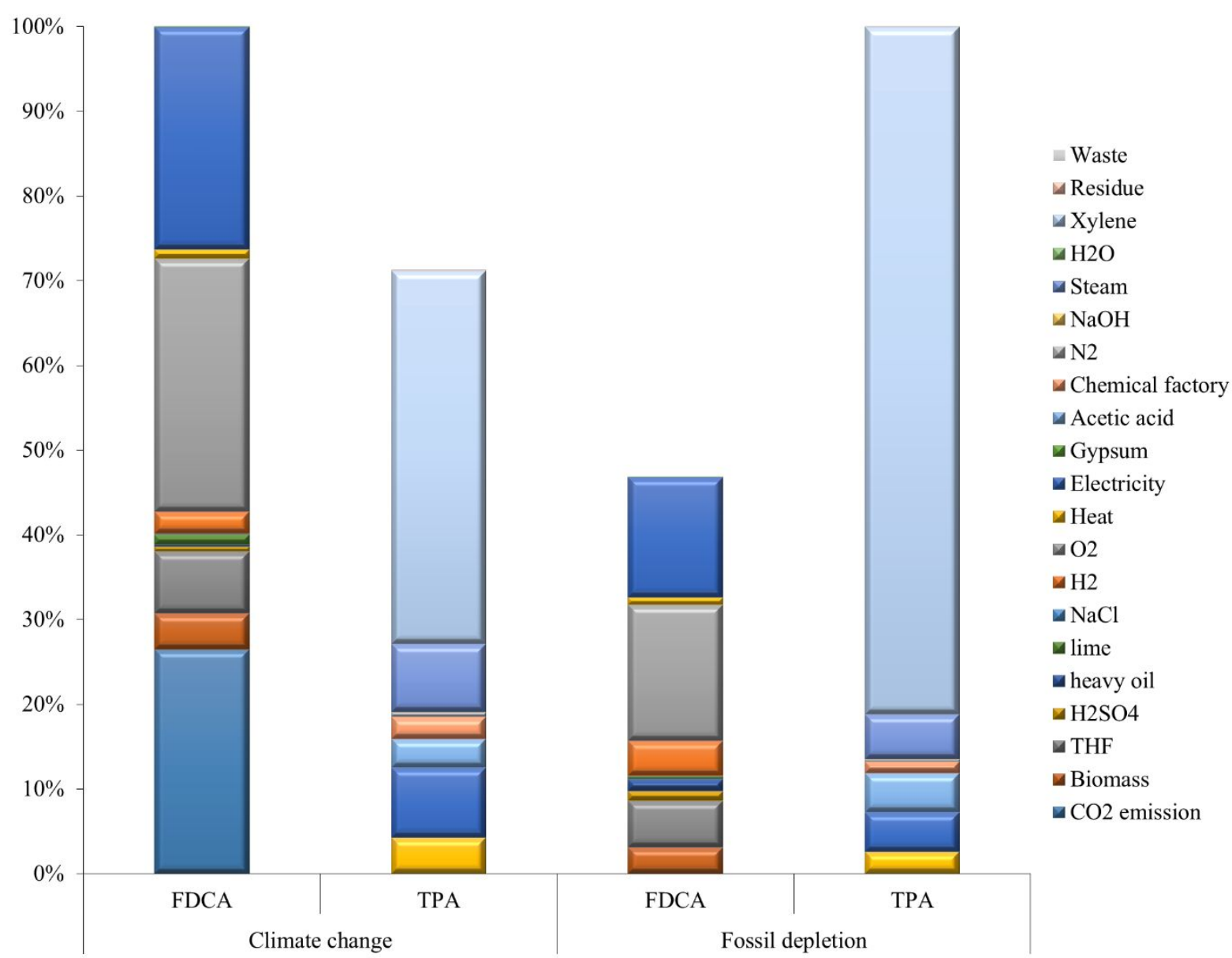

Figure S5. Comparison of main contributors to two environmental impact indicators (CC and FD) between the productions of petroleum-derived TPA and biomass-derived FDCA.

Table S11. Environmental impact results of the two productions for main contributors.

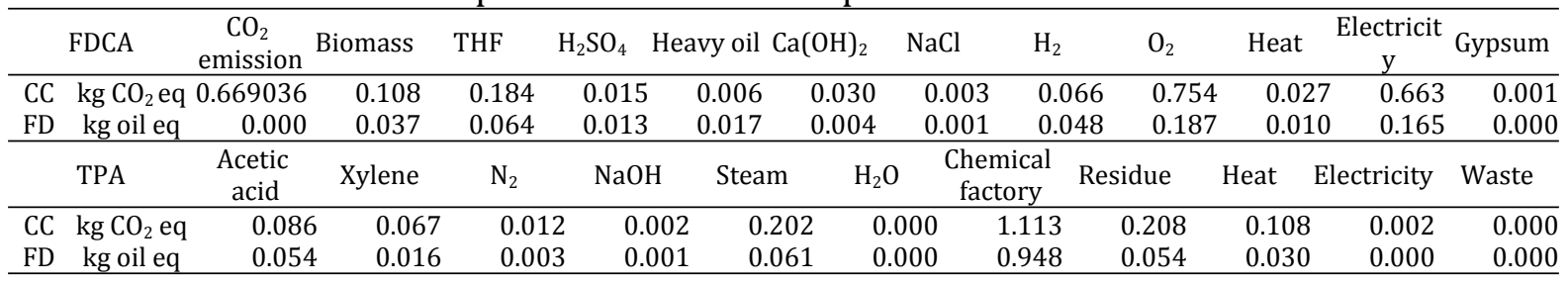




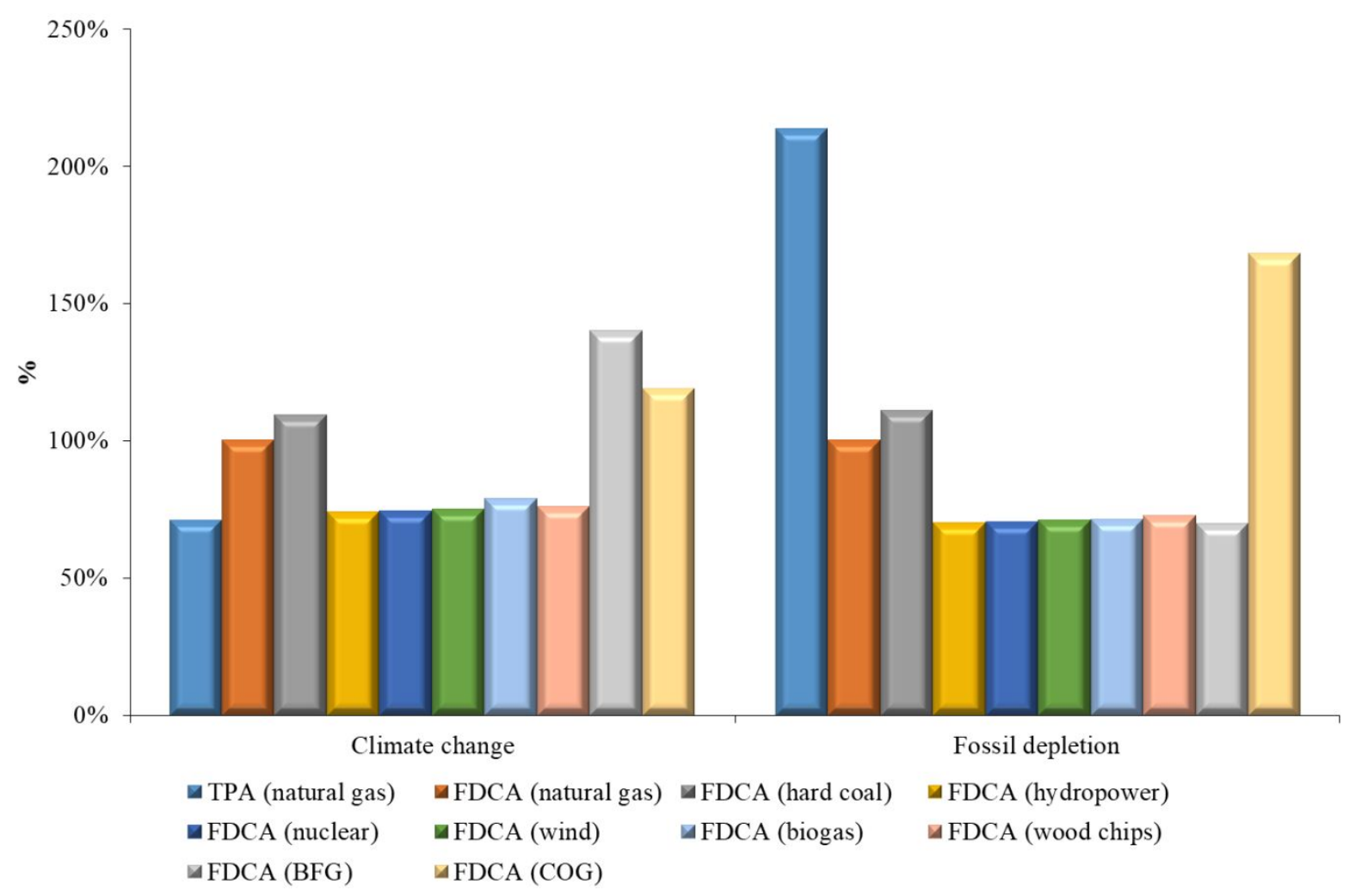

Figure S6. Comparison of the environmental impact results in the variation of feedstocks and technologies used in the biomass-derived FDCA production.

Table S12. Environmental impact results (CC and FD) in the variation of feedstocks and technologies used in the biomass-derived FDCA production.

\begin{tabular}{|c|c|c|c|c|c|c|c|c|c|c|}
\hline & TPA (NG) & $\begin{array}{c}\text { FDCA } \\
(\mathrm{NG})\end{array}$ & $\begin{array}{l}\text { FDCA } \\
\text { (hard } \\
\text { coal) }\end{array}$ & $\begin{array}{l}\text { FDCA } \\
\text { (hydro) }\end{array}$ & $\begin{array}{c}\text { FDCA } \\
\text { (nuclear) }\end{array}$ & $\begin{array}{l}\text { FDCA } \\
\text { (wind) }\end{array}$ & $\begin{array}{c}\text { FDCA } \\
\text { (biogas) }\end{array}$ & $\begin{array}{l}\text { FDCA } \\
\text { (wood } \\
\text { chips) }\end{array}$ & $\begin{array}{l}\text { FDCA } \\
\text { (BFG) }\end{array}$ & $\begin{array}{l}\text { FDCA } \\
\text { (COG) }\end{array}$ \\
\hline $\mathrm{CC} \operatorname{kg~CC}$ & 1.800 & 2.525 & 2.760 & 1.866 & 1.872 & 1.890 & 1.991 & 1.916 & 3.535 & 3.004 \\
\hline FD kg oil eq & 1.167 & 0.546 & 0.606 & 0.382 & 0.384 & 0.388 & 0.390 & 0.397 & 0.382 & 0.918 \\
\hline
\end{tabular}




\section{References}

1. Alonso DM, Hakim SH, Zhou S, Won W, Hosseinaei O, Tao J, Garcia-Negron V, Motagamwala AH, Mellmer MA, Huang K, Houtman CJ, Labbe N, Harper DP, Maravelias CT, Runge T, Dumesic JA. Increasing the revenue from lignocellulosic biomass: maximizing feedstock utilization. Science Advances 2017;3:e1603301. https://doi.org/10.1126/sciadv.1603301.

2. Davis R, Tao L, Scarlata C, Tan ECD, Ross J, Lukas J, Sexton D. Process design and economics for the conversion of lignocellulosic biomass to hydrocarbons. National Renewable Energy Laboratory Report 2015;NREL/TP-5100-62498. https://doi.org/10.2172/1176746.

3. He J, Huang K, Barnett KJ, Krishna SH, Alonso DM, Brentzel ZJ, Burt SP, Walker T, Banholzer WF, Maravelias CT, Hermans I, Dumesic JA, Huber G.W. New catalytic strategies for $\alpha, \omega$-diols production from lignocellulosic biomass. Faraday Discussion 2017;202:247-267. https://doi.org/10.1039/C7FD00036G.

4. Dorris CC, Lu E, Park S, Toro FH. High-purity oxygen production using mixed ionic-electronic conducting sorbent, Working paper, University of Pennsylvania 2016.

5. Han J, Sen SM, Luterbacher JS, Alonso DM, Dumesic JA, Maravelias CT. Process systems engineering studies for the synthesis of catalytic biomass-to-fuels strategies. Computers \& Chemical Engineering 2015;81:57-69. https://doi.org/10.1016/j.compchemeng.2015.04.0 07.

6. Dutta A, Sahir A, Tan E, Humbird D, Snowden-Swan LJ, Meyer P, Ross J, Sexton D, Yap R, Lukas J. Process design and economics for the conversion of lignocellulosic biomass to hydrocarbon fuels. National Renewable Energy Laboratory Report 2015;NREL/TP-5100-62455. https://doi.org/10.2172/1238302.

7. Han J, Sen SM, Alonso DM, Dumesic JA, Maravelias CT. A strategy for the simultaneous catalytic conversion of hemicellulose and cellulose from lignocellulosic biomass to liquid transportation fuels. Green Chemistry 2014;16:653-661. https://doi.org/10.1039/ C3GC41511B.

8. Brentzel ZJ, Barnett KJ, Huang K, Maravelias CT, Dumesic JA, Huber GW. Chemicals from biomass: combining ring-opening tautomerization and hydrogenation reactions to produce 1,5-pentanediol from furfural. ChemSusChem 2017;10:1351-1355. https://doi.org/ 10.1002/cssc.201700178.

9. Motagamwala AH, Won W, Senser C, Alonso DM, Maravelias CT, Dumesic JA. Toward biomassderived renewable plastics: production of 2,5-furandicarboxylic acid from fructose. Science Advances 2018;4:eaap9772. https://doi.org/10.1126/sciadv.aap9722. 